\title{
A short proof of the Strong Normalization of Classical Natural Deduction with Disjunction
}

\author{
René David and Karim NOUR \\ LAMA - Equipe de Logique \\ Université de Chambéry \\ 73376 Le Bourget du Lac \\ e-mail: \{david,nour\}@univ-savoie.fr
}

\begin{abstract}
We give a direct, purely arithmetical and elementary proof of the strong normalization of the cut-elimination procedure for full (i.e. in presence of all the usual connectives) classical natural deduction.
\end{abstract}

\section{Introduction}

This paper gives a direct proof of the strong normalization of the cut-elimination procedure for full propositional classical logic. By full we mean that all the connectives $(\rightarrow, \wedge$ and $\vee$ ) and $\perp$ (for the absurdity) are considered as primitive and they have their intuitionistic meaning. As usual, the negation is defined by $\neg A=A \rightarrow \perp$.

It is well known that, when the underlying logic is the classical one (i.e. the absurdity rule is allowed) these connectives are redundant since, for example, $\vee$ and $\wedge$ can be coded by using $\rightarrow$ and $\perp$. From a logical point of view, considering the full logic is thus somehow useless. However, from the computer science point of view, considering the full logic is interesting because, by the so-called Curry-Howard isomorphism, formulas can be seen as types for functional programming languages and correct programs can be extracted from proofs. For that reason various systems have been studied in the last decades (see, for example, [2, 4, 5, 12, 14, 15, 17, 22]) both for intuitionistic and classical logic. The connectives $\wedge$ and $\vee$ have a functional counter-part $(\wedge$ corresponds to a product and $\vee$ to a co-product, i.e. a case of $)$ and it is thus useful to have them as primitive.

Until very recently (see the introduction of [7] for a brief history), no proof of the strong normalization of the cut-elimination procedure was known for full logic. In [7], de Groote gives such a proof by using a CPS-style transformation from full classical logic to intuitionistic logic with $\rightarrow$ as the only connective, i.e. the simply typed $\lambda$-calculus. A very elegant and direct proof of the strong normalization of the full logic is given in [11] but only the intuitionistic case is given.

We give here another proof of de Groote's result. This proof is based on a proof of the strong normalization of the simply typed $\lambda$-calculus due to the first author (see [8]) which, itself, is a simplification of the one given by Matthes in [11]. After this paper had been written we were told by Curien and some others that this kind of technique was already present in van Daalen (see [27]) and Levy (see [13]). The same idea is used in [10] to give a short proof of the strong normalization of the simply typed $\lambda \mu$-calculus of [17]. Apart the fact that this proof is direct (i.e. uses no translation into an other system whose strong normalization is known) and corresponds to the intuition (the main argument of the proof is an induction on the complexity of the cut-formula) we believe that our technique is quite general and may be used in other circumstances. A crucial lemma of our proof is used in [16] to give a semantical proof of the strong normalization. Finally [9] uses 
the same technique to give an elementary proof of the strong normalization of a typed $\lambda$-calculus with explicit substitutions which, from the logical point of view, correspond to explicit cuts and weakenings.

\section{The typed system}

We code proofs by using a set of terms (denoted $\mathcal{T}$ ) which extends the $\lambda \mu$-terms of Parigot [17] and is given by the following grammar where $x, y, \ldots$ are (intuitionistic) variables and $a, b, \ldots$ are (classical) variables:

$$
\begin{gathered}
\mathcal{T}::=x|\lambda x \mathcal{T}|(\mathcal{T} \mathcal{E})|\langle\mathcal{T}, \mathcal{T}\rangle| \omega_{1} \mathcal{T}\left|\omega_{2} \mathcal{T}\right| \mu a \mathcal{T} \mid(a \mathcal{T}) \\
\mathcal{E}::=\mathcal{T}\left|\pi_{1}\right| \pi_{2} \mid[x . \mathcal{T}, y \cdot \mathcal{T}]
\end{gathered}
$$

The meaning of the new constructors is given by the typing rules of figure 1 below where $\Gamma$ is a context, i.e. a set of declarations of the form $x: A$ and $a: \neg A$ where $x$ is an intuitionistic variable, $a$ is a classical variable and $A$ is a formula.

Note that, since we only are concerned with the logical point of view, we should only consider typed terms, i.e. use a $\lambda$-calculus à la Church. However, for the simplicity of notation, the set of terms has been given in an untyped formalism i.e. we use a $\lambda$-calculus à la Curry.

$$
\begin{gathered}
\frac{\Gamma_{1} \vdash M: A \quad \Gamma_{2} \vdash N: B}{\Gamma, x: A \vdash x: A} a x \quad \frac{\Gamma \vdash M: A_{1} \wedge A_{2}}{\Gamma_{1}, \Gamma_{2} \vdash\langle M, N\rangle: A \wedge B} \wedge_{i} \quad \frac{\Gamma \vdash\left(M \pi_{i}\right): A_{i}}{\Gamma} \wedge_{e} \\
\frac{\Gamma, x: A \vdash M: B}{\Gamma \vdash \lambda x M: A \rightarrow B} \rightarrow_{i} \quad \frac{\Gamma_{1} \vdash M: A \rightarrow B \quad \Gamma_{2} \vdash N: A}{\Gamma_{1}, \Gamma_{2} \vdash(M N): B} \rightarrow_{e} \\
\frac{\Gamma \vdash M: A_{i}}{\Gamma \vdash \omega_{i} M: A_{1} \vee A_{2}} \vee_{i} \\
\frac{\Gamma \vdash M: A_{1} \vee A_{2} \quad \Gamma_{1}, x_{1}: A_{1} \vdash N_{1}: C \quad \Gamma_{2}, x_{2}: A_{2} \vdash N_{2}: C}{\Gamma, \Gamma_{1}, \Gamma_{2} \vdash\left(M\left[x_{1} \cdot N_{1}, x_{2} \cdot N_{2}\right]\right): C} \vee_{e} \\
\frac{\Gamma, a: \neg A \vdash M: \perp}{\Gamma \vdash \mu a M: A} a b s_{i} \quad \frac{\Gamma, a: \neg A \vdash M: A}{\Gamma \vdash(a M): \perp} a b s_{e}
\end{gathered}
$$

Figure 1.

This coding is essentially the same as in [1] and [7]. We have adopted the notations of [1] which are also used by [11]: what is written $\pi_{i} M$ in [7] is written $\left(M \pi_{i}\right)$ here and $\delta\left(M, x_{1} . N_{1}, x_{2} . N_{2}\right)$ in [7] is written $\left(M\left[x_{1} \cdot N_{1}, x_{2} . N_{2}\right]\right)$ here. These notations have the advantage of making the permutative and classical reduction rules more uniform and thus simplifies the proofs.

The cut-elimination procedure corresponds to the reduction rules given below. There are three kinds of cuts.

Logical cuts: they appear when the introduction of a connective $(\rightarrow, \wedge$ and $\vee)$ is immediately followed by its elimination. The corresponding rules are:

- $(\lambda x M N) \triangleright M[x:=N]$

- $\left(\left\langle M_{1}, M_{2}\right\rangle \pi_{i}\right) \triangleright M_{i}$

- $\left(\omega_{i} M\left[x_{1} \cdot N_{1}, x_{2} . N_{2}\right]\right) \triangleright N_{i}\left[x_{i}:=M\right]$

Permutative cuts : they appear when the elimination rule of the disjunction is followed by the elimination rule of a connective. They are considered as cuts because a logical cut may be hidden by the $\vee_{e}$ rule. Considering these cuts is necessary to get the sub-formula property. The corresponding rule is: 
- $\left(M\left[x_{1} . N_{1}, x_{2} . N_{2}\right] \varepsilon\right) \triangleright\left(M\left[x_{1} \cdot\left(N_{1} \varepsilon\right), x_{2} .\left(N_{2} \varepsilon\right)\right]\right)$

Classical cuts : they appear when the classical rule is followed by the elimination rule of a connective. The corresponding rule is:

- $(\mu a M \varepsilon) \triangleright \mu a M\left[a:={ }^{*} \varepsilon\right]$ where $M\left[a:={ }^{*} \varepsilon\right]$ is obtained by replacing each sub-term of $M$ of the form $(a N)$ by $(a(N \varepsilon))$.

Notation 2.1 Let $M$ be in $\mathcal{E}$.

1. $M \triangleright M^{\prime}$ means that $M$ reduces to $M^{\prime}$ by using one step of the reduction rules given above. As usual, $\triangleright^{+}\left(\right.$resp. $\left.\triangleright^{*}\right)$ is the transitive (resp. reflexive and transitive) closure of $\triangleright$.

2. $M$ is strongly normalizable (this is denoted by $M \in S N$ ) if there is no infinite sequence of $\triangleright$ reductions.

Remark If $M=\left[y_{1} \cdot N_{1}, y_{2} \cdot N_{2}\right], M \triangleright M^{\prime}$ means that $M^{\prime}$ is either $\left[y_{1} \cdot N_{1}^{\prime}, y_{2} \cdot N_{2}\right]$ or $\left[y_{1} \cdot N_{1}, y_{2} \cdot N_{2}^{\prime}\right]$ where $N_{1} \triangleright N_{1}^{\prime}$ or $N_{2} \triangleright N_{2}^{\prime}$. It is thus clear that $M \in S N$ iff $N_{1}, N_{2} \in S N$.

The following result is straightforward.

Lemma 2.1 (Subject reduction) If $\Gamma \vdash M: A$ and $M \triangleright^{*} N$ then $\Gamma \vdash N: A$.

The goal of this paper is the proof of theorem 2.1 below.

Theorem 2.1 Every typed term is strongly normalizable.

The proof is an immediate corollary of theorem 4.1: if $M, N \in S N$, then $M[x:=$ $N] \in S N$.

The proof of theorem 4.1 uses a characterization of strongly normalizable (theorem 3.1): a term is in $S N$ iff its arguments and head reduct (see definition 3.3) are in $S N$. This theorem needs another result (theorem 3.2) which is, intuitively, very clear but whose formal proof needs some work.

The main difficulties are the following:

- The first one is minor: in the $\lambda$-calculus, each term has a unique head, either a head variable or a head redex. Due to the connective $\wedge$, this is no longer true here and a term may have both a head variable and a head redex. This is treated by showing that it is enough to consider only the simple terms (see definition 3.1).

- The second one is crucial and due to the presence of critical pairs such as $\left(\mu a M\left[y_{1} . N_{1}, y_{2} . N_{2}\right] \varepsilon\right)$. We can choose as head redex either the classical one or the permutative one. If we choose the classical one, the proof of theorem 3.1 will be easy but the proof of theorem 4.1 does not work because, in the rule $\vee_{e}$, the type of the main hypothesis has nothing to do with the type of its conclusion. We thus have to choose, as head redex, the permutative one but then, theorem 3.1 needs the difficult theorem 3.2. For the same reason (the rule $\vee_{e}$ ), the proof of theorem 4.1 needs a rather complex induction: we use a 5 -tuple of integers. Note that E. Tahhan Bittar [3] has given a proof of the strong normalization of the sequent calculus by using essentially the same 5 -tuple of integers.

Remark It is also for simplicity of proofs that, in the totality of this section, we only consider typed terms and thus, for example, that terms such as $\left(\lambda x M\left[x_{1} \cdot N_{1}, x_{2} . N_{2}\right]\right)$ are not allowed because they, obviously, cannot be typed since the type of $\lambda x M$ must be an implication. Actually, theorems 3.1 and 3.2 would also be true for untyped terms i.e. even if terms as $\left(\lambda x M\left[x_{1} . N_{1}, x_{2} . N_{2}\right]\right)$ were allowed and its proof will be essentially the same since such a term is strongly normalizable iff $M, N_{1}, N_{2}$ also are strongly normalizable. 


\section{Characterization of strongly normalizable terms}

Definition 3.1 1. A term $M$ is simple if $M$ either is a variable or an application.

2. The set of contexts is given by the following grammar:

$$
C:=*_{i}|\lambda x C| \omega_{i} C\left|\left\langle C_{1}, C_{2}\right\rangle\right| \mu a C
$$

3. If $C$ is a context with holes $*_{1}, \ldots, *_{n}$ and $M_{1}, \ldots, M_{n}$ are terms, $C\left[M_{1}, \ldots, M_{n}\right]$ is the term obtained by replacing each $*_{i}$ by $M_{i}$.

Lemma 3.1 Each term $M$ can be uniquely written as $C\left[M_{1}, \ldots, M_{n}\right]$ where $C$ is a context and $M_{1}, \ldots, M_{n}$ are simple terms.

Proof By induction on $M$.

Lemma 3.2 Let $C$ be a context and $M_{1}, \ldots, M_{n}$ be terms. Then $C\left[M_{1}, \ldots, M_{n}\right] \in$ $S N$ iff $M_{1}, \ldots, M_{n} \in S N$.

Proof By induction on $C$.

Definition 3.2 (and notation) $A$ (possibly empty) sequence $\vec{N}=N_{1}, \ldots, N_{n}$ of elements of $\mathcal{E}$ is nice if each $N_{i} \in \mathcal{T} \cup\left\{\pi_{1}, \pi_{2}\right\}$ except possibly for $i=n$. If $M$ is a term, $\left(M N_{1} \ldots N_{n}\right)$ will be denoted as $(M \vec{N})$.

Lemma 3.3 (and definition) Let $M$ be a simple term.

1. Then $M$ can be uniquely written as one of the cases of the figure below where $\vec{T}=T_{1}, \ldots, T_{n}$ is a nice sequence and, in case (4) and (5), $\varepsilon \vec{T}$ is also nice, i.e. if $\varepsilon=\left[y_{1} \cdot N_{1}, y_{2} . N_{2}\right]$ then $\vec{T}$ is empty.

2. The set of arguments of $M$ (denoted as $\arg (M)$ ) and the head of $M$ (denoted as $h d(M)$ ), either a redex or a variable, are defined by the figure below.

3. The head reduct of $M$ (denoted as hred $(M)$ ) is the term obtained by reducing, if any, the head redex of $M$.

\begin{tabular}{|c|l|c|c|}
\hline & $M$ & $h d(M)$ & $\arg (M)$ \\
\hline 0 & $(x \vec{T})$ or $(a T)$ & $x$ or $a$ & $\left\{T_{1}, \ldots, T_{n}\right\}$ or $T$ \\
\hline 1 & $(\lambda x N O \vec{T})$ & $(\lambda x N O)$ & $\{O\}$ \\
\hline 2 & $\left(\left\langle N_{1}, N_{2}\right\rangle \pi_{i} \vec{T}\right)$ & $\left(\left\langle N_{1}, N_{2}\right\rangle \pi_{i}\right)$ & $\left\{N_{1}, N_{2}\right\}$ \\
\hline 3 & $\left(\omega_{i} N\left[x_{1} \cdot O_{1}, x_{2} \cdot O_{2}\right]\right)$ & $M$ & $\left\{N, O_{1}, O_{2}\right\}$ \\
\hline 4 & $(\mu a N \varepsilon \vec{T})$ & $(\mu a N \varepsilon)$ & $\{\varepsilon\}$ \\
\hline 5 & $\left(N\left[x_{1} \cdot O_{1}, x_{2} \cdot O_{2}\right] \varepsilon \vec{T}\right)$ & $\left(N\left[x_{1} . O_{1}, x_{2} \cdot O_{2}\right] \varepsilon\right)$ & $\emptyset$ \\
\hline
\end{tabular}

Proof Since $M$ is simple, and for trivial typing reasons, it looks like either (a) $(x \vec{S})$ or $(a S)$ or $(\mathrm{b})(\lambda x N O \vec{S})$ or $(\mathrm{c})\left(\left\langle N_{1}, N_{2}\right\rangle \pi_{i} \vec{S}\right)$ or $(\mathrm{d})\left(\omega_{i} N\left[x_{1} . O_{1}, x_{2} . O_{2}\right] \vec{S}\right)$ or (e) $(\mu a N \varepsilon \vec{S})$. If $\vec{S}$ is empty the result is clear.

Otherwise, assume first $\vec{S}$ is nice. The cases (a), (b) and (c) are clear. Case (d) gives (5). Case (e) gives (5) if $\varepsilon=\left[y_{1} \cdot N_{1}, y_{2} . N_{2}\right]$ or (4) otherwise.

Assume finally $\vec{S}$ is not nice. Then $\vec{S}$ can be written as $\overrightarrow{S_{1}}\left[y_{1} \cdot N_{1}, y_{2} \cdot N_{2}\right] \overrightarrow{S_{2}}$ where $\overrightarrow{S_{2}}$ is nice and non empty. It is then easy to see that, in all cases, this gives (5) where $\overrightarrow{S_{2}}=\varepsilon \vec{T}$.

For uniqueness, check easily (by looking wether $\vec{T}$ has an $\left[y_{1} \cdot N_{1}, y_{2} . N_{2}\right]$ or not) that if $M$ is in case 0 to 4 it cannot also be in case 5 . 
Theorem 3.1 Let $M$ be a simple term. If $M$ has an head redex, then $M \in S N$ iff $\arg (M) \subset S N$ and hred $(M) \in S N$. Otherwise, $M \in S N$ iff $\arg (M) \subset S N$.

Proof The case of an head variable is trivial. Case 1 of the figure of lemma 3.3 is done as follows. Since $h r e d(M) \in S N, N$ and $\vec{T}$ are in $S N$. Thus and since $\vec{T}$ is nice, an infinite reduction of $M$ must look like: $M \triangleright^{*}\left(\lambda x N_{1} O_{1} \overrightarrow{T_{1}}\right) \triangleright\left(N_{1}[x:=\right.$ $\left.\left.O_{1}\right] \overrightarrow{T_{1}}\right) \triangleright \ldots$. The contradiction comes from the fact (see lemma 3.4 below) that $\operatorname{hred}(M) \triangleright^{*}\left(N_{1}\left[x:=O_{1}\right] \overrightarrow{T_{1}}\right)$. Cases 2, 3, 4 are similar.

Case 5 is theorem 3.2 below.

Lemma 3.4 Let $M, N \in \mathcal{T}$. Assume $M \triangleright M^{\prime}$ and $N \triangleright N^{\prime}$. Let $\sigma$ (resp. $\sigma^{\prime}$ ) be either $[x:=N]$ or $\left[a:={ }^{*} N\right]$ (resp. $\left[x:=N^{\prime}\right]$ or $\left.\left[a:={ }^{*} N^{\prime}\right]\right)$. Then $M[\sigma] \triangleright M^{\prime}[\sigma]$ and $M[\sigma] \triangleright^{*} M\left[\sigma^{\prime}\right]$.

Proof Straightforward.

Theorem 3.2 Assume the sequence $\varepsilon \vec{V}$ is nice and $S_{2}=\left(N\left[x_{1} .\left(N_{1} \varepsilon\right), x_{2} .\left(N_{2} \varepsilon\right)\right]\right.$ $\vec{V}) \in S N$. Then $S_{1}=\left(M\left[x_{1} . N_{1}, x_{2} . N_{2}\right] \varepsilon \vec{V}\right) \in S N$.

Proof See section 5 .

\section{Proof of theorem 2.1}

By induction on $M$. The cases $x, \lambda x N,\langle N, O\rangle, \omega_{i} N,(a N)$ and $\mu a N$ are immediate. The last case is $M=(N \varepsilon)=(x \varepsilon)[x:=N]$ where $x$ is a fresh variable and the result follows from the induction hypothesis and theorem 4.1 below.

Definition 4.1 Let $M$ be a term. Then, $\operatorname{cxty}(M)$ is the number of symbols occurring in $M$ and, if $M \in S N, \eta(M)$ is the length of the longest reduction of $M$.

In lemma 4.1 and theorem 4.1 below, $\sigma$ denotes a substitution of the form $\left[x_{i}:=N_{i} / i=1 \ldots n\right]$, i.e. we substitute only intuitionistic variables.

Lemma 4.1 Let $M$ be a simple term with an head redex and $\sigma$ be a substitution. Then, $h d(M[\sigma])=h d(M)[\sigma], \arg (M[\sigma])=\arg (M)[\sigma]$ and $h r e d(M[\sigma])=$ $\operatorname{hred}(M)[\sigma]$.

Proof Immediate.

Theorem 4.1 Let $M \in S N$ be a term and $\sigma$ be a substitution. Assume that the substituted variables all have the same type and, for all $x, \sigma(x) \in S N$. Then $M[\sigma] \in S N$.

Proof

The proof is by induction on $(\operatorname{lgt}(\sigma)), \eta(M), \operatorname{cxty}(M), \eta(\sigma), \operatorname{cxty}(\sigma))$ where $\operatorname{lgt}(\sigma)$ is the number of connectives in the type of the substituted variables and $\eta(\sigma)$ (resp. $\operatorname{cxty}(\sigma))$ is the sum of the $\eta(N)($ resp. $\operatorname{cxty}(N))$ for the $N$ that are actually substituted, i.e. for example if $\sigma=[x:=N]$ and $x$ occurs $n$ times in $M$, then $\eta(\sigma)=n . \eta(N)$ and $\operatorname{cxty}(\sigma)=n . \operatorname{cxty}(N)$. The induction hypothesis will be abbreviated as $I H$.

By the $I H$ and lemmas 3.2 and 3.1 we may assume that $M$ is simple. Consider then the various cases of lemma 3.3.

- If $M$ has an head redex: by lemma 4.1 and the $I H, \arg (M[\sigma]) \subseteq S N$ since for each $N \in \arg (M), \operatorname{cxty}(N)<\operatorname{cxty}(M)$. By lemma 4.1, hred $(M[\sigma])=$ $\operatorname{hred}(M)[\sigma]$ and thus, since $\eta(\operatorname{hred}(M))<\eta(M), \operatorname{hred}(M[\sigma]) \in S N$ follows from the $I H$. 
- Otherwise, if the head variable is a classical variable or an intuitionistic variable not in the domain of $\sigma$, the result is trivial.

- Otherwise, i.e $M=(x \vec{T})$

- If $h d(M[\sigma])$ is a variable, the result is trivial.

- If $h d(M[\sigma])=h d(\sigma(x))$ : let $M^{\prime}=z \vec{T}$ where $z$ is a fresh variable and $\sigma^{\prime}$ be the substitution defined as follows $\sigma^{\prime}(z)=\operatorname{hred}(\sigma(x))$ and, for the variables $y$ occurring in $\vec{T}, \sigma^{\prime}(y)=\sigma(y)$. Then, $\operatorname{hred}(M[\sigma])=M^{\prime}\left[\sigma^{\prime}\right]$ and thus, by the $I H$, $h r e d(M[\sigma]) \in S N$ since $\eta\left(\sigma^{\prime}\right)<\eta(\sigma)$.

- Otherwise, the head redex has been created by the substitution. The various cases are:

1. $M=(x O \vec{S})$ and $\sigma(x)=\lambda y N$. By the $I H, \arg (M[\sigma]) \subseteq S N$ and thus, by theorem 3.1, we have to show that $P=(N[y:=O[\sigma]] \overrightarrow{S[\sigma]}) \in S N$. By the $I H$, $(z \overrightarrow{S[\sigma]}) \in S N$ and since $\operatorname{lgt}(O[\sigma])<\operatorname{lgt}(\lambda y N), N[y:=O[\sigma]] \in S N$. Thus $P=(z \overrightarrow{S[\sigma]})[z:=N[y:=O[\sigma]]] \in S N$ since $\operatorname{lgt}(N[y:=O[\sigma]])<\operatorname{lgt}(\lambda y N)$.

2. $M=\left(x \pi_{i} \vec{S}\right)$ and $\sigma(x)=\left\langle N_{1}, N_{2}\right\rangle$ or $M=\left(x\left[x_{1} \cdot M_{1}, x_{2} \cdot M_{2}\right]\right)$ and $\sigma(x)=$ $\omega_{i} N$. The proof is similar.

3. $M=\left(x\left[x_{1} \cdot M_{1}, x_{2} \cdot M_{2}\right]\right)$ and $\sigma(x)=\mu a N$. By the $I H, \arg (M[\sigma]) \subseteq S N$ and thus (by theorem 3.1) we have to show $P=\mu a N\left[a:=^{*}\left[x_{1} . P_{1}, x_{2} . P_{2}\right]\right] \in S N$ where, for $i=1,2, P_{i}=M_{i}[\sigma]$. Since $\operatorname{cxty}\left(M_{i}\right)<\operatorname{cxty}(M)$, the fact that $P_{i} \in S N$ follows from the $I H$. The result is thus a particular case of the claim below.

Claim Let $P_{1}, P_{2}, T \in S N$ and $a_{1}, \ldots, a_{n}$ be variables of type $\neg(A \vee B)$. Let $T[\tau]$ denotes $T\left[a_{i}:={ }^{*}[P] / i=1 \ldots n\right]$ where $[P]$ is an abbreviation for $\left[x_{1} . P_{1}, x_{2} . P_{2}\right]$. Then $T[\tau] \in S N$.

Proof By induction on $(\eta(T), \operatorname{cxty}(T))$. We may assume that $T$ is simple. Consider the various cases of lemma 3.3.

- If $T$ has an head redex, the result follows immediately from $I H$ and lemma 4.1.

- Otherwise and if the head variable of $T$ is not in $\tau$, the result is trivial.

- Otherwise and because of the type of the $a_{i}, T=(a V)$ where $V \in \mathcal{T}$. It is thus enough to prove that $(V[\tau][P]) \in S N$ and, for that, it is enough to show that its head reduct $Q \in S N$. The various cases are:

$-V=\omega_{i} W$ and $Q=P_{i}\left[x_{i}:=W[\tau]\right]$. By the $I H, W[\tau] \in S N$ since $\operatorname{cxty}(W)<\operatorname{cxty}(T)$ and thus, since $\operatorname{lgt}(W)<\operatorname{lgt}(N), Q \in S N$ follows from the main $I H$ (recall we are "inside" the proof of theorem $4.1, \operatorname{type}(W)=A$ or type $(W)=B$ and type $(N)=A \vee B)$.

$-V=\mu b W$ and $Q=\mu b W[\tau]\left[b:={ }^{*}[P]\right]=\mu b W\left[\tau^{\prime}\right]$ where $\tau^{\prime}=$ $\tau \cup\left[b:={ }^{*}[P]\right]$. Since $\operatorname{cxty}(W)<\operatorname{cxty}(T)$, the result follows from the IH.

- $V=(W \varepsilon)$ and $\varepsilon$ is not in the form $\left[x_{1} \cdot W_{1}, x_{2} \cdot W_{2}\right]$. Then, the head redex of $(V[\tau][P])$ must come from $V$ and $Q=\left(V^{\prime}[\tau][P]\right)$ for some $V^{\prime}$ such that $V \triangleright V^{\prime}$. Let $T^{\prime}=\left(a V^{\prime}\right)$. Since $\eta\left(T^{\prime}\right)<\eta(T)$, $T^{\prime}[\tau] \in S N$. But $T^{\prime}[\tau] \triangleright Q$ and thus $Q \in S N$.

- $V=\left(W\left[x_{1} . W_{1}, x_{2} . W_{2}\right]\right)$ and $Q=\left(W[\tau]\left[x_{1} \cdot\left(W_{1}[\tau][P]\right), x_{2} .\left(W_{2}[\tau][P]\right)\right]\right)$. Let $T_{j}=\left(a W_{j}\right)$. Since $\operatorname{cxty}\left(T_{j}\right)<\operatorname{cxty}(T)$, by the $I H, T_{j}[\tau] \in S N$ 
and thus $\left(W_{j}[\tau][P]\right) \in S N$ since $T_{j}[\tau] \triangleright\left(W_{j}[\tau][P]\right)$. By the $I H$, since $\operatorname{cxty}(W)<\operatorname{cxty}(T), W[\tau] \in S N$. By theorem 3.1, it is thus enough to show that $Q^{\prime}=\operatorname{hred}(Q) \in S N$.

If $h d(Q)$ comes from $W$, the result follows from the $I H$. Otherwise, the various cases are:

* $W=\omega_{i} W^{\prime}$ and $Q^{\prime}=\left(W_{i}[\tau][P]\right)\left[x_{i}:=W^{\prime}[\tau]\right]$. Let $T^{\prime}=$ $\left(a W_{i}\left[x_{i}:=W^{\prime}\right]\right)$. Then $T=\left(a\left(\omega_{i} W^{\prime}\left[x_{1} \cdot W_{1}, x_{2} \cdot W_{2}\right]\right)\right) \triangleright T^{\prime}$.

By the $I H, T^{\prime}[\tau] \in S N$ and the result follows from the fact that $T^{\prime}[\tau] \rightarrow\left(W_{i}[P]\right)\left[x_{i}:=W^{\prime}\right][\tau]=Q^{\prime}$.

* If $W=\mu b W^{\prime}$ or $W=\left(W^{\prime}\left[x_{1} . W_{1}^{\prime}, x_{2} \cdot W_{2}^{\prime}\right]\right)$ : the proof is similar.

4. $M=(x \varepsilon \vec{T}), \varepsilon \neq\left[x_{1} \cdot M_{1}, x_{2} \cdot M_{2}\right]$ and $\sigma(x)=\mu a N$. We prove exactly as in case 3 that $(\mu a N \varepsilon[\sigma]) \in S N$. To prove that $M[\sigma] \in S N$, it is enough to use the same trick as in case 1: $M[\sigma]=(z \vec{T}[\sigma])[z:=(\mu a N \varepsilon[\sigma])]$ where $z$ is a fresh variable and the $I H$ gives the result since $\operatorname{lgt}(z)<\operatorname{lgt}(x)$.

5. $M=\left(x\left[x_{1} \cdot M_{1}, x_{2} \cdot M_{2}\right]\right)$ and $\sigma(x)=\left(N_{3}\left[y_{1} \cdot N_{1}, y_{2} \cdot N_{2}\right]\right)$. By theorem 3.1, it is enough to show $P=\left(N_{3}\left[y_{1} \cdot\left(N_{1}[P]\right), y_{2} \cdot\left(N_{2}[P]\right)\right]\right) \in S N$ where, for $i=1,2, P_{i}=M_{i}[\sigma]$ and $\left(N_{i}[P]\right)$ is a notation for $\left(N_{i}\left[x_{1} \cdot P_{1}, x_{2} \cdot P_{2}\right]\right)$. Let $M^{\prime}=\left(z\left[x_{1} \cdot M_{1}, x_{2} \cdot M_{2}\right]\right)$ where $z$ be a fresh variable. For $i=1,2$, let $\sigma_{i}=\sigma \cup$ $\left[z:=N_{i}\right]$. By the $I H, M^{\prime}\left[\sigma_{i}\right] \in S N$ since $\eta\left(\sigma^{\prime}\right) \leq \eta(\sigma)$ and $\operatorname{cxty}\left(\sigma^{\prime}\right)<\operatorname{cxty}(\sigma)$. Then $\left(N_{i}[P]\right) \in S N$ since $M^{\prime}\left[\sigma_{i}\right] \triangleright\left(N_{i}[P]\right)$. By theorem 3.1, it is thus enough to show that $Q=\operatorname{hred}(P) \in S N$.

If $h d(P)$ comes from $W$, the result follows from the $I H$. Otherwise, the various cases are:

- $N_{3}=\omega_{i} N_{3}^{\prime}$ and $Q=\left(N_{i}\left[x_{i}:=N_{3}^{\prime}\right][P]\right)$. Let $M^{\prime}=\left(z\left[x_{1} \cdot M_{1}, x_{2} \cdot M_{2}\right]\right)$ where $z$ is a fresh variable and $\sigma^{\prime}=\sigma \cup\left\{\left[z:=N_{i}\left[x_{i}:=N_{3}^{\prime}\right]\right\}\right.$. Then $Q=M^{\prime}\left[\sigma^{\prime}\right] \in S N$ since $\eta\left(\sigma^{\prime}\right) \leq \eta(\sigma)$ and $\operatorname{cxty}\left(\sigma^{\prime}\right)<\operatorname{cxty}(\sigma)$.

- $N_{3}=\mu a N_{3}^{\prime}$ or $N_{3}=\left(Q_{3}\left[y_{1} \cdot Q_{1}, y_{2} \cdot Q_{2}\right]\right)$. The proof is similar.

6. If $M=(x \varepsilon \vec{T}), \varepsilon \neq\left[x_{1} \cdot M_{1}, x_{2} \cdot M_{2}\right]$ and $\sigma(x)=\left(N_{3}\left[x_{1} \cdot N_{1}, x_{2} \cdot N_{2}\right]\right)$. We prove exactly as in case 5 that $\left(N_{3}\left[x_{1} \cdot N_{1}, x_{2} . N_{2}\right] \varepsilon[\sigma]\right) \in S N$. To prove that $M[\sigma] \in S N$, it is enough to use the same trick as in case 1: $M[\sigma]=$ $(z \vec{T}[\sigma])\left[z:=\left(N_{3}\left[x_{1} \cdot N_{1}, x_{2} . N_{2}\right] \varepsilon[\sigma]\right)\right]$ where $z$ is a fresh variable and the $I H$ gives the result since $\operatorname{lgt}(z)<\operatorname{lgt}(x)$.

\section{$5 \quad$ Proof of theorem 3.2}

The idea of the proof is the following: we show that an infinite reduction of $S_{1}$ can be translated into an infinite reduction of $S_{2}$. These reductions are the same except that, in $S_{1}, \varepsilon$ can be far away from the $N_{i}$. We mark $\varepsilon$ and the $N_{i}$ to keep their trace. This gives the set of marked terms $\mathcal{T}^{\prime}$ of definition 5.1. The correct terms of definition 5.3 intuitively are the marked terms for which each marked $N_{i}$ knows who is the corresponding marked $\varepsilon$. Concretely, being correct is a sufficient condition to ensure that a reduction in the marked $S_{1}$ can be translated to the corresponding $S_{2}$.

The main difficulty of the proof consists in writing precise definitions. The proofs of the lemmas consist in easy but tedious verifications.

Important remark. The proof is uniform in the sequence $\varepsilon \vec{V}$. In definition 5.1 below, we implicitly assume the following: if we are proving theorem 3.2 for $\varepsilon \in \mathcal{T}$ 
(resp. $\varepsilon=\pi_{i}, \varepsilon=\left[y_{1} . M_{1}, y_{2} . M_{2}\right]$ ) then, in the sub-terms of the form $\circ_{\varepsilon^{\prime}}$, we necessarily have $\varepsilon^{\prime} \in \mathcal{T}$ (resp. $\varepsilon^{\prime}=\pi_{i}, \varepsilon^{\prime}=\left[y_{1} . Q_{1}, y_{2} . Q_{2}\right]$ ). Note that we could also assume that $\varepsilon^{\prime}$ is a reduct of $\varepsilon$ but this does not really matter for the proof. However, in the case $\varepsilon=\left[y_{1} . P_{1}, y_{2} . P_{2}\right]$, since the sequence $\varepsilon \vec{V}$ is nice $\vec{V}$ is empty and this must appear in the proof. We will do the proof only for $\varepsilon \in \mathcal{T}$ or $\varepsilon=\pi_{i}$. The proof for the case $\varepsilon=\left[y_{1} . P_{1}, y_{2} . P_{2}\right]$ is essentially the same: we just have to add an third condition in definition 5.3 and check in the lemmas that this condition is preserved. This new condition is given in the final remark of this section.

Definition 5.1 1. Let $\mathcal{T}^{\prime}$ be the set of terms obtained from $\mathcal{T}$ by adding new constructors: $\diamond_{N}$ and $\circ_{\varepsilon}$ where $N \in \mathcal{T}$ and $\varepsilon \in \mathcal{E}$ are closed.

2. The reduction rules for $\mathcal{T}^{\prime}$ are the ones of $\mathcal{T}$ plus the following:

- If $N \triangleright N^{\prime}$ then $\diamond_{N} \triangleright \diamond_{N^{\prime}}$ and $\circ_{N} \triangleright \circ_{N^{\prime}}$.

- $\left(\diamond_{N} \circ_{\varepsilon}\right) \triangleright(N \varepsilon)$.

3. Let be the congruence defined by the following reduction rules:

- $\left(M\left[x_{1} \cdot N_{1}, x_{2} \cdot N_{2}\right] \circ_{\varepsilon}\right) \triangleright\left(M\left[x_{1} \cdot\left(N_{1} \circ_{\varepsilon}\right), x_{2} \cdot\left(N_{2} \circ_{\varepsilon}\right)\right]\right)$

- $\left(\mu a M \circ_{\varepsilon}\right) \triangleright \mu a M\left[a:={ }^{*} \circ_{\varepsilon}\right]$

Comments

An element of $\mathcal{T}^{\prime}$ is a term in $\mathcal{T}$ where some sub-terms have been replaced by terms as $\diamond_{N}$ or $\circ_{\varepsilon}$ where $N \in \mathcal{T}$ and $\varepsilon \in \mathcal{E}$ and, in particular, have no sub-terms as $\diamond_{N^{\prime}}$ or $\circ_{\varepsilon^{\prime}}$. It is assumed, in the definition, that the $N$ and $\varepsilon$ occurring in $\diamond_{N}$ or $\circ_{\varepsilon}$ are closed. In fact, they are allowed to have free variables (both intuitionistic and classical) but it is assumed that these variables will never be captured and thus act as constants.

Definition 5.2 Let $M \in \mathcal{T}^{\prime}$.

1. $M$ is acceptable iff $M=\diamond_{N}$ or $M=\mu a M_{1}$ and, for each sub-term of $M$ of the form $(a N), N$ is acceptable or $M=\left(N\left[x_{1} . N_{1}, x_{2} \cdot N_{2}\right]\right)$ and $N_{1}, N_{2}$ are acceptable.

2. If $M$ is acceptable, the set st $(M)$ of terms is defined by: st $\left(\diamond_{N}\right)=\left\{\diamond_{N}\right\}$, $s t\left(\mu a M_{1}\right)=\cup\left\{\operatorname{st}(S) /(a S)\right.$ sub-term of $\left.M_{1}\right\}$ and $\operatorname{st}\left(\left(N\left[x_{1} \cdot N_{1}, x_{2} \cdot N_{2}\right]\right)\right)=$ $\operatorname{st}\left(N_{1}\right) \cup \operatorname{st}\left(N_{2}\right)$.

Lemma 5.1 Let $M \in \mathcal{T}^{\prime}$ be an acceptable term.

1. If $\sigma$ is a substitution either of the form $[x:=N]$ or $\left[a:={ }^{*} N\right]$, then $M[\sigma]$ is acceptable and $\operatorname{st}(M[\sigma])=\operatorname{st}(M)$.

2. If $M \triangleright M^{\prime}$, then $M^{\prime}$ is acceptable and $\operatorname{st}\left(M^{\prime}\right) \subseteq \operatorname{st}(M)$.

Proof By induction on $M$. (1) trivial. For (2) use (1).

Definition 5.3 A term $M \in \mathcal{T}^{\prime}$ is correct if the following conditions hold.

1. Each occurrence of a term of the form $\circ_{\varepsilon}$ appears as $\left(U \circ_{\varepsilon}\right)$ for some acceptable term $U$.

2. For each sub-term of $M$ of the form $\diamond_{N}$ there is a sub-term (necessarily unique) of the form $\left(U \circ_{\varepsilon}\right)$ such that $\diamond_{N}$ belongs to st $(U)$. The corresponding $\varepsilon$ is denoted as eps $(N)$ 


\section{Examples}

- Assume $M, N, O, P, \varepsilon$ are closed terms. Then $A=\left(M\left[x_{1} \diamond_{N}, x_{2} . \diamond_{O}\right] \circ_{\varepsilon} P\right)$ is correct.

- Assume $M, N, O, P, Q, R, S, \varepsilon_{1}, \varepsilon_{2}$ are closed terms. Then $B=$ $\left(M\left[x_{1} \cdot\left(N\left[y_{1} \cdot \diamond_{O}, y_{2} \cdot \mu a P\right] \circ_{\varepsilon_{1}}\right), x_{2} \cdot\left(\mu b\left(b \mu c\left(c\left(Q\left[z_{1} \cdot \mu d R, z_{2} \cdot \diamond_{S}\right]\right)\right)\right){ }_{\varepsilon_{2}}\right)\right]\right)$ is correct.

Lemma 5.2 If $M$ is correct and $M \triangleright M^{\prime}$, then $M^{\prime}$ is correct.

Proof Let $\left(U \circ_{\varepsilon}\right)$ be a sub-term of $M$. A reduction can be, either in $\varepsilon$ or in $U$ or between $U$ and $\varepsilon$ or, finally, above $\left(U \circ_{\varepsilon}\right)$. Since $U$ is acceptable and by using lemma 5.1 it is easy to check that, in each case the conditions of correctness are still satisfied.

Lemma 5.3 Let $M$ be a correct term.

1. $M$ has no sub-term of the form $\left(O \diamond_{N}\right)$.

2. If $\left(\diamond_{N} O\right)$ is a sub-term of $M$, then $O=o_{\varepsilon}$ for some $\varepsilon$.

Proof Otherwise, let $\left(U \circ_{\varepsilon}\right)$ be the sub-term such that $\diamond_{N} \in s t(U)$. The result follows easily from the fact that $U$ is acceptable.

Definition 5.4 Let $M \in \mathcal{T}^{\prime}$.

1. $T_{1}(M)$ is the term obtained by replacing $\diamond_{N}$ by $N$ and $\circ_{\varepsilon}$ by $\varepsilon$.

2. If $M$ is a sub-term of a correct term, $T_{2}(M)$ is the term obtained by replacing each occurrence of $\left(U \circ_{\varepsilon}\right)$ by $U^{\prime}$ where $U^{\prime}$ is obtained from $U$ by replacing each occurrence of $\diamond_{N}$ such that $\varepsilon=\operatorname{eps}(N)$ is a sub-term of $M$ by $(N \varepsilon)$.

\section{Comments and examples}

1. If $M$ itself is correct, $T_{2}(M) \in \mathcal{T}$. Otherwise, some $\diamond_{N}$ that are related to a $\circ_{\varepsilon}$ outside $M$ are not replaced. We need this more general definition for the proof of lemma 5.4 .

2. If $M$ is correct, $T_{1}(M) \triangleright^{*} T_{2}(M)$. More precisely $T_{2}(M)=T_{1}\left(M^{\prime}\right)$ where $M^{\prime}$ is the normal form of $M$ for the rules $\boldsymbol{~}$. Since we will not use this result, we do not prove it.

3. Let $A, B$ be the terms of the previous example. Then

$T_{1}(A)=\left(M\left[x_{1} . N, x_{2} . O\right] \varepsilon P\right)$ and $T_{2}(A)=\left(M\left[x_{1} .(N \varepsilon), x_{2} \cdot(O \varepsilon)\right] P\right)$.

$T_{1}(B)=\left(M\left[x_{1} \cdot\left(N\left[y_{1} \cdot O, y_{2} \cdot \mu a P\right] \varepsilon_{1}\right), x_{2} \cdot\left(\mu b\left(b \mu c\left(c\left(Q\left[z_{1} \cdot \mu d R, z_{2} \cdot S\right]\right)\right)\right) \varepsilon_{2}\right)\right]\right)$ and $T_{2}(B)=\left(M\left[x_{1} \cdot\left(N\left[y_{1} \cdot\left(O \varepsilon_{1}\right), y_{2} \cdot \mu a P\right]\right), x_{2} \cdot \mu b\left(b \mu c\left(c\left(Q\left[z_{1} \cdot \mu d R\right.\right.\right.\right.\right.\right.$, $\left.\left.\left.\left.\left.\left.z_{2} \cdot\left(S \varepsilon_{2}\right)\right]\right)\right)\right)\right]\right)$.

Lemma 5.4 Let $M \in \mathcal{T}^{\prime}$ be correct. If $T_{1}(M) \triangleright N$, there is a correct term $M^{\prime}$ such that $M \triangleright^{+} M^{\prime}$ and $T_{1}\left(M^{\prime}\right)=N$.

Proof Let $R$ be the redex that has been reduced. By lemma 5.3, the only cases to consider are:

- There is a redex $S$ in $M$ such that $R=T_{1}(S)$. The result follows then from lemma 5.3.

- There is a sub-term of $M$ of the form $\diamond_{N}$ or $\circ_{\varepsilon}$, such that $R$ is a sub-term of $N$ or $\varepsilon$. The result is then trivial.

- Finally, $R=\left(T_{1}(U) \varepsilon\right)$ where $\left(U \circ_{\varepsilon}\right)$ is a sub-term of $M$, the result follows from the fact that $U$ is acceptable. 
Lemma 5.5 Let $P=(M O)$ be a sub-term of a correct term. Assume $O \neq \circ_{\varepsilon}$ and $P \triangleright P^{\prime}$ by reducing the redex $P$. Then, $T_{2}(P) \triangleright^{*} T_{2}\left(P^{\prime}\right)$.

Proof It is, for example, straightforward to check that, if $M=\lambda x M_{1}$, then $T_{2}(P)=\left(\lambda x T_{2}\left(M_{1}\right) T_{2}(O)\right)$ and $T_{2}\left(P^{\prime}\right)=T_{2}\left(M_{1}\right)\left[x:=T_{2}(O)\right]$. The other cases are similar.

Lemma 5.6 Assume $M=\left(N \circ_{\varepsilon}\right)$ is a sub-term of a correct term. Then,

- $T_{2}(M)=T_{2}\left(\left(T_{2}(N) \circ_{\varepsilon}\right)\right)$.

- If $N$ has no sub-terms of the form $\circ_{\varepsilon^{\prime}}$ and $N \triangleright N^{\prime}$ then, $T_{2}(M) \triangleright^{*} T_{2}\left(\left(N^{\prime} \circ_{\varepsilon}\right)\right)$.

Proof Straightforward.

Lemma 5.7 Let $M$ is a sub-term of a correct term. If $M \triangleright M^{\prime}$, then $T_{2}(M) \triangleright^{*}$ $T_{2}\left(M^{\prime}\right)$. Moreover, if $T_{2}(M)=T_{2}\left(M^{\prime}\right)$, then $M-M^{\prime}$.

Proof By induction on $(n b(M)$, cxty $(M))$ where $n b(M)$ is the number of subterms of the form $\circ_{\varepsilon}$ in $M$. The only non trivial case is $M=(N O)$.

- Assume $O \neq o_{\varepsilon}$. If $M^{\prime}=\left(N^{\prime} O\right)$ where $N \triangleright N^{\prime}$ or $M^{\prime}=\left(N O^{\prime}\right)$ where $O \triangleright O^{\prime}$, the result is trivial. Otherwise, $M$ itself is the reduced redex and the result comes from lemma 5.5.

- Assume $O=\circ_{\varepsilon}$. If $M$ itself is the reduced redex then $T_{2}(M)=T_{2}\left(M^{\prime}\right)$ and $M \triangleright M^{\prime}$. If $M^{\prime}=\left(N \circ_{\varepsilon^{\prime}}\right)$ where $\varepsilon \triangleright \varepsilon^{\prime}$, the result is trivial. Otherwise, i.e. $M^{\prime}=\left(N^{\prime} \circ_{\varepsilon}\right)$ where $N \triangleright N^{\prime}$. If $n b(N)=0$, the result follows from lemma 5.6(2). Otherwise, by the induction hypothesis, $T_{2}(N) \triangleright^{*} T_{2}\left(N^{\prime}\right)$ and the result comes from lemma 5.6.

Lemma 5.8 Let $M \in \mathcal{T}^{\prime}$ be a correct term. Then $M$ is strongly normalizable for the reduction.

Proof If $M$ is correct, let $l g(M)$ be the sum of the length of the path (i.e. the number of nodes in the tree representing $M)$ relating the $\diamond_{N}$ to the corresponding ${ }^{\circ}$. It is easy to see that, if $M \triangleright M^{\prime}$, then $\lg \left(M^{\prime}\right)<\lg (M)$.

\section{Proof of theorem 3.2}

Assume $S_{2} \in S N$ and $S_{1} \notin S N$. Let $\left(U_{i}\right)$ be a sequence of terms such that $U_{0}=S_{1}$ and, for each $i, U_{i} \triangleright U_{i+1}$. Let $M=\left(N\left[x_{1} \cdot \diamond_{N_{1}}, x_{2} . \diamond_{N_{2}}\right] \circ_{\varepsilon} \vec{V}\right)$. By using lemma 5.4, we get a sequence of correct terms $M_{i}$ such that, for each $i, M_{i} \triangleright^{+} M_{i+1}$ and $T_{1}\left(M_{i}\right)=U_{i}$. By lemma 5.7, $T_{2}\left(M_{i}\right) \triangleright^{*} T_{2}\left(M_{i+1}\right)$. Since $S_{2}=T_{2}(M) \in S N$, there is an $i_{0}$ such that, for $i \geq i_{0}, T_{2}\left(M_{i}\right)=T_{2}\left(M_{i+1}\right)$ and thus, by lemma 5.7, $M_{i} \triangleright^{+} M_{i+1}$. This contradicts lemma 5.8 .

\section{Remark.}

Assume that, in theorem $3.2, \varepsilon=\left[y_{1} \cdot Q_{1}, y_{2} \cdot Q_{2}\right]$. If $\vec{V}$ were not empty, the proof of lemma 5.4 would not work because a redex could be created by the transformation $T_{1}$. Here is an example: let $M=\left(\begin{array}{lll}P & \circ_{\varepsilon} & V\end{array}\right)$ be correct and assume $T_{1}(M)=$ $\left(P\left[y_{1} \cdot Q_{1}, y_{2} \cdot Q_{2}\right] V\right) \triangleright N=\left(P\left[y_{1} \cdot\left(Q_{1} V\right), y_{2} \cdot\left(Q_{2} V\right)\right]\right)$. There is no way to find $M^{\prime}$ such that $M \triangleright M^{\prime}$ and $T_{1}\left(M^{\prime}\right)=N$ because $\left(P \circ_{\varepsilon} V\right)$ is not a redex.

We do not know wether theorem 3.2 remains true if the sequence $\varepsilon \vec{V}$ is not nice: to prove it, $\left(P \circ_{\varepsilon} V\right)$ should then be considered as a redex but $\mathcal{T}^{\prime}$ becomes much more complicated. Since $\varepsilon \vec{V}$ is nice, it is simpler to add a new condition in the definition 5.3 of correctness to ensure that this situation (of the creation of a redex by the transformation $T_{1}$ ) does not appear. This condition is the following: 
3. $M$ is good wrt the set of all its sub-terms of the form $\left(U \circ_{\varepsilon}\right)$.

where, if $E$ is a subset of $\mathcal{T}^{\prime}, M$ is good wrt to $E$ is defined by: $M \in E$ or $M=\mu a N$ and for each occurrence of $(a N)$ in $M, N$ is good wrt to $E$ or $M=$ $\left(N\left[x_{1} . N_{1}, x_{2} . N_{2}\right]\right)$ and $N_{1}, N_{2}$ are good wrt to $E$.

This condition implies in particular that, in a correct term, there is no sub-term of the form $\left(N \circ_{\varepsilon} U\right)$ and thus that lemma 5.4 remains valid. It is not difficult to check that the other lemmas remain also valid.

\section{References}

[1] Y. Andou. Church-Russer property of simple reduction for full first-order classical natural deduction. Annals of Pure and Applied logic 119 (2003) 225-237.

[2] F. Barbanera and S. Berardi A symmetric lambda-calculus for classical program extraction. In M. Hagiya and J.C. Mitchell, editors, Proceedings of theoretical aspects of computer software, TACS'94. LNCS (789), pp. 495-515. Springer Verlag, 1994.

[3] E. T. Bittar. Strong Normalisation Proofs for Cut-Elimination in Gentzen's Sequent Calculi. Logic, Algebra and Computer Science, 46, Banach Center Publications, pp. 179-225, 1999.

[4] R. Constable and C. Murthy Finding computational content in classical proofs. In G. Huet and G. Plotkin, editors, Logical Frameworks, pp. 341-362, Cambridge University Press, 1991.

[5] J.-Y. Girard A new constructive logic: classical logic. MSCS (1), pp. 255-296, 1991.

[6] P. de Groote. On the strong normalization of natural deduction with permutation-conversions. In 10th International Conference on Typed Lambda Calculi and Applications, TLCA'95. LNCS (902) pp. 201-215. Springer Verlag, 1995.

[7] P. de Groote. Strong normalization of classical natural deduction with disjunction. In 5th International Conference on typed lambda calculi and applications, TLCA'01. LNCS (2044), pp. 182-196. Springer Verlag, 2001.

[8] R. David. Normalization without reducibility. Annals of Pure and Applied Logic (107), p. 121-130, 2001.

[9] R. David and B. Guillaume. Strong normalization of the typed $\lambda_{w s}$-calculus. Accepted in the CSL'03 colloquium.

[10] R. David and K. Nour. A short proof of the strong normalization of the simply typed $\lambda \mu$-calculus. To appear in Schedae Informaticae vol.12, 2003.

[11] F. Joachimski and R. Matthes. Short proofs of normalization for the simplytyped lambda-calculus, permutative conversions and Gödel's T. Archive for Mathematical Logic 42, pp 59-87 (2003).

[12] J.-L. Krivine. Classical logic, storage operators and 2nd order lambda-calculus. Annals of Pure and Applied Logic (68), pp. 53-78, 1994.

[13] J.J. Levy. Rductions correctes et optimales dans le lambda-calcul. $\mathrm{PhD}$ thesis Paris 7, 1978. 
[14] C.R. Murthy. An evaluation semantics for classical proofs. In Proceedings of the sixth annual IEEE symposium on logic in computer science, pp. 96-107, 1991.

[15] C.R. Murthy. A computational analysis of Girard's translation and LC. In Proceedings of the seventh annual IEEE symposium on logic in computer science, pp. 90-101, 1992 .

[16] K. Nour and K. Saber. A semantical proof of the strong normalization theorem of full propositionnal classical natural deduction. Manuscript 2003.

[17] M. Parigot $\lambda \mu$-calculus: An algorithm interpretation of classical natural deduction. Lecture Notes in Artificial Intelligence (624), pp. 190-201. Springer Verlag 1992.

[18] M. Parigot. Proofs of strong normalization for second order classical natural deduction. Journal of Symbolic Logic, 62 (4), pp. 1461-1479, 1997.

[19] D. Prawitz. Natural deduction, a proof-theoretical study. Almqvist \& Wiksell, Stockholm, 1965.

[20] D. Prawitz. Ideas and results in proof-theory. In J.E. Fenstad, editor, Proceedings of the second scandinavian logic symposium, pp. 237-309. North-Holland, 1971.

[21] D. Pym and E. Ritter. On the semantics of classical disjunction. Journal of Pure and Applied Algebra, To appear.

[22] N.J. Rehof and M.H. Sorensen. The $\lambda_{\Delta}$-calculus. In M. Hagiya and J.C. Mitchell, editors, Proceedings of the international symposium on theoretical aspects of computer software, TACS'94, LNCS (789), pp. 516-542. Springer Verlag, 1994.

[23] E. Ritter, D. Pym and L. Wallen. On the intuitionistic force of classical search. Theoretical Computer Science (232), pp. 299-333, 2000.

[24] E. Ritter, D. Pym and L. Wallen. Proof-terms for classical and intuitionistic resolution. Journal of logic and computation, 10 (2), pp. 173-207, 2000.

[25] J. Seldin. On the proof theory of the intermediate logic MH. Journal of symbolic logic, 51 (3), pp. 626-647, 1986.

[26] G. Stalmarck. Normalization theorems for full first-order classical natural deduction. Journal of symbolic logic, 56 (1), pp. 129-149, 1991.

[27] D. van Daalen The language theory of Automath. PhD Thesis. Eindhoven 1977. 